\title{
Payer Costs for Inpatient Treatment of Pathologic Fracture, Surgery to Bone, and Spinal Cord Compression Among Patients with Multiple Myeloma or Bone Metastasis Secondary to Prostate or Breast Cancer
}

\author{
Arie Barlev, PharmD, MS; Xue Song, PhD; Boris Ivanov, MS; \\ Vidya Setty, MPH, MBA; and Karen Chung, PharmD, MS
}

\begin{abstract}
BACKGROUND: Patients with bone metastasis secondary to prostate or breast cancer or multiple myeloma are predisposed to skeletal-related events (SREs), such as surgery or radiation to the bone, pathologic fracture, and spinal cord compression. Inpatient costs of these and other SREs represent an estimated $49 \%-59 \%$ of total costs related to SREs. However, information on payer costs for hospitalizations associated with SREs is limited, especially for costs associated with specific SREs by tumor type.
\end{abstract}

OBJECTIVE: To examine costs from a payer perspective for SRE-associated hospitalizations among patients with multiple myeloma or bone metastasis secondary to prostate or breast cancer.

METHODS: Patients with SRE hospitalizations were selected from the MarketScan commercial and Medicare databases (January 1, 2003, through June 30, 2009). Sampled patients had at least 2 medical claims with primary or secondary ICD-9-CM diagnosis codes for prostate cancer, breast cancer, or multiple myeloma and at least 1 subsequent hospitalization with principal diagnosis or procedure codes indicating bone surgery, pathologic fracture, or spinal cord compression. For patients with prostate cancer or breast cancer, a diagnosis code for bone metastasis was also required. If secondary diagnoses or procedure codes for SREs were present in the claim, they were used to more precisely identify the type of SRE for which the patient was treated, resulting in 3 mutually exclusive categories: spinal cord compression with or without pathologic fracture and/or surgery to the bone; pathologic fracture with or without surgery to the bone; and only surgery to the bone. Related readmissions within 30 days of a previous SRE-associated hospitalization date of discharge were excluded to minimize the risk of underestimating costs. Mean health plan payments per hospitalization, measured as net reimbursed amounts paid by the health plan to a hospital after subtracting patient copayments and deductibles, were analyzed by cancer type and type of SRE.

RESULTS: A total of 555 patients contributed 572 hospitalizations that met the study criteria for prostate cancer, 1,413 patients contributed 1,542 hospitalizations for breast cancer, and 1,361 patients contributed 1,495 hospitalizations for multiple myeloma. The mean age range was 61 to 72 years, and the mean length of stay per admission was 5.9 to 11.6 days across the 3 tumor types. The ranges of mean health plan payment per hospital admission across tumor types were $\$ 43,691-\$ 59,854$ for spinal cord compression, with or without pathologic fracture and/or surgery to the bone; $\$ 22,390-\$ 26,936$ for pathologic fracture without spinal cord compression, with or without surgery to the bone; and $\$ 31,016-\$ 42,094$ for surgery to the bone without pathologic fracture or spinal cord compression.

CONCLUSIONS: The inpatient costs associated with treating SREs are significant from a payer perspective. Our study used a systematic process for patient selection and mutually exclusive categorization by SRE type and provides a per episode estimate of the inpatient financial impact of cancerrelated SREs assessed in this study from a third-party payer perspective.

J Manag Care Pharm. 2010;16(9):693-702

Copyright $\odot 2010$, Academy of Managed Care Pharmacy. All rights reserved.

\section{What is already known about this subject}

- Two published studies have estimated the mean health care costs associated with treatment of skeletal-related events (SRE) in patients with cancer and bone metastasis, providing a broad picture of estimated costs in both the inpatient and outpatient settings.

- A study by Lage et al. (2008) of patients with prostate cancer estimated that the mean cost of SREs per person, measured as dollars billed to health plans, was $\$ 12,469$ in 2006 dollars for treatment of pathologic fracture, spinal cord compression, surgery to bone, and radiation to bone in the year after initial SRE diagnosis.

- Delea et al. (2006) studied patients with breast cancer and estimated an average per SRE treatment cost over 60 months (mean follow-up 13.8 months) of $\$ 13,940$ in 2002 dollars for pathologic fracture, spinal cord compression, radiation or surgery to bone, hypercalcemia, or use of opioid analgesics.

- In both studies, $49 \%$ or more of the health care costs were incurred in the inpatient setting, but the breakdown of inpatient costs by type of SRE was not provided. These studies report costs over time rather than costs per SRE episode. The majority of patients in both studies experienced only 1 SRE (78\% in the prostate cancer study and $61 \%$ in the breast cancer study).

\section{What this study adds}

- The current study reports costs for inpatient treatment of SREs from the payer perspective, measured as net payment to the facility after subtracting patient copayments and deductibles, by type of SRE across 3 tumor types: prostate cancer, breast cancer, and multiple myeloma.

- Mean inpatient cost estimates for the 3 tumor types ranged from $\$ 31,016$ to $\$ 42,094$ for bone surgery, from $\$ 22,390$ to $\$ 26,936$ for pathologic fracture, and from $\$ 43,691$ to $\$ 59,854$ for spinal cord compression. 


\section{What this study adds (continued)}

- As the focus of this study was on inpatient payer costs of SREassociated treatment, radiation to bone was not included as an independent SRE.

- The results of this study will be the first published inpatient cost estimates for SREs in patients with multiple myeloma in the United States.

$\mathrm{B}$ one is a common site for the spread of several malignancies as its nutrient-rich environment provides a favorable soil for colonizing tumor cells., When tumor cells metastasize to bone, they are thought to secrete cytokines and growth factors that induce osteoblasts (bone-forming cells) to release the protein RANK ligand (RANKL). In turn, RANKL promotes the formation and survival of osteoclasts (bone-resorbing cells), which, when activated, cause local bone destruction in the direct area of the tumor metastasis. Key growth factors are released from the bone breakdown that may promote proliferation, metastasis, and survival of tumor cells. Thus, a "vicious cycle" of tumor expansion and bone destruction resorption is perpetuated. ${ }^{3}$ Bone metastases can result in significant skeletal complications known as skeletalrelated events (SREs), such as pathologic fracture, spinal cord compression, or need for surgery or radiation to bone. ${ }^{4-8}$ Nearly $70 \%$ of patients with metastatic prostate or breast cancer experience metastases to the bone, and up to $95 \%$ of patients with multiple myeloma experience osteolytic bone lesions, which may lead to SREs. ${ }^{9-12}$

Breast and prostate cancers are the most prevalent cancers in the United States, with 1 in 8 women developing breast cancer and 1 in 6 men developing prostate cancer during their lifetimes. ${ }^{13}$ Additionally, virtually all cases of myeloma involve bone destruction. ${ }^{14}$ Given these facts, the economic burden that SREs associated with these cancers can place on health systems may be substantial. Treatments that can reduce or prevent SREs may reduce the economic burden of these skeletal complications. Intravenous bisphosphonates, primarily zoledronic acid (Zometa; Novartis), are effective for preventing SREs. ${ }^{15}$ Denosumab (Amgen), a RANKL inhibitor, has effectively delayed and reduced the occurrence of SREs compared with zoledronic acid in ongoing clinical trials. ${ }^{16-18}$ To better assess the potential cost-offsets gained by these therapies, it can be helpful to understand the cost burden that each type of SRE places on the health care system.

Treatment of SREs occurs in both inpatient and outpatient settings. Inpatient treatment represented an estimated 49\%$59 \%$ of total SRE costs in previous research. ${ }^{19,20}$ This analysis focuses on third-party payer costs for treating SRE episodes in the inpatient setting by type of SRE. Previously published cost-
of-SRE studies do not identify the attributable costs of individual types of SREs. A study of patients with advanced prostate cancer conducted by Lage et al. (2008) reported that mean hospital costs as dollars billed per patient in the year after the initial diagnosis of an SRE was $\$ 12,469$, with the highest costs for radiation therapy $(\$ 5,930)$, pathologic fracture $(\$ 3,179)$, then bone surgery $(\$ 2,218) .{ }^{19}$ Another study of patients with advanced breast cancer conducted by Delea et al. (2006) reported billed average costs per SRE over 60 months (mean follow-up 13.8 months) among a limited subset of patients with SREs matched to patients without SREs and found that total medical care costs were $\$ 48,173$ (95\% confidence interval $[C I]=\$ 19,068-\$ 77,684)$ greater in patients with SREs versus patients without SREs $(P=0.001){ }^{20}$ Since inpatient costs are a significant component of the total cost of care of SREs, focusing this analysis on the inpatient setting enabled us to quantify the individual components that contribute to the per episode inpatient costs of SREs. Future studies will quantify the contribution of outpatient care and costs per SRE episode in these patient populations.

\section{Methods}

\section{Data Sources}

Data for this study were obtained from the MarketScan Commercial Claims \& Encounter (commercial) database and the Medicare Supplemental \& Coordination of Benefit (Medicare) database from Thomson Reuters. The databases are constructed based on claims and enrollment data provided by 138 medium to large employers and 13 health plans from across the United States. Data include medical claims for health care services performed in both the inpatient and outpatient settings and enrollment data, including member demographic information and eligibility and benefits data. The medical claims files include service dates, provider reimbursement amounts, patient copayment and deductible amounts, and Medicare coordination of benefit amounts where appropriate. The medical claims and enrollment data are linked to outpatient prescription drug claims through the use of unique enrollee identifiers. Together, the commercial and Medicare databases captured information on approximately 34.6 million covered lives in the working population and 2.5 million retirees in the Medicare population in 2008. The Medicare database profiles the health care experience of retirees and includes the Medicare-covered portion of payment, the employer-paid portion, and any patient out-of-pocket expenses. The database is compliant with the Health Insurance Portability and Accountability Act (HIPAA). Because this study did not involve the collection, use, or transmittal of individually identifiable data, Institutional Review Board (IRB) review or approval was not sought. 


\section{Sample Selection}

The study sample consisted of patients with multiple myeloma, prostate cancer, or breast cancer with claims between January 1, 2003, and June 30, 2009. We selected SRE hospitalizations for patients who had (a) 2 or more claims at least 30 days apart with primary or secondary diagnosis of multiple myeloma (International Classification of Diseases, Ninth Revision, Clinical Modification [ICD-9-CM] 203.0x), prostate cancer (ICD-
9-CM 185.xx, V10.46), or breast cancer (ICD-9-CM 174.xx, 175.xx, V10.3) and (b) hospitalizations for one or more SREs as principal diagnosis (ICD-9-CM) or principal procedure (ICD9-CM or Current Procedural Terminology [CPT]) at any time. In addition, at least 1 non-rule-out bone metastasis diagnosis (ICD-9-CM 198.5) before or on the date of admission was required for patients with prostate cancer or breast cancer.

SRE hospitalizations were further categorized using 


\section{Payer Costs for Inpatient Treatment of Pathologic Fracture, Surgery to Bone, and Spinal Cord Compression Among Patients with Multiple Myeloma or Bone Metastasis Secondary to Prostate or Breast Cancer}

\section{TABLE 1 Demographic Characteristics and Hospitalization Discharge Status}

\begin{tabular}{|c|c|c|c|c|c|c|}
\hline \multirow{3}{*}{$\begin{array}{l}\text { Number of hospitalizations } \\
\text { Number of unique patients } \\
\text { b }\end{array}$} & \multicolumn{2}{|c|}{ Prostate Cancer } & \multicolumn{2}{|c|}{ Breast Cancer } & \multicolumn{2}{|c|}{ Multiple Myeloma } \\
\hline & \multicolumn{2}{|c|}{572} & \multicolumn{2}{|c|}{1,542} & \multicolumn{2}{|c|}{1,495} \\
\hline & 555 & $(100.0 \%)$ & 1,413 & $(100.0 \%)$ & 1,361 & $(100.0 \%)$ \\
\hline \multirow[t]{2}{*}{ Mean [SD] age } & 72.1 & [11.6] & 61.3 & {$[12.8]$} & 65.4 & {$[12.5]$} \\
\hline & $\mathbf{n}$ & $(\%)$ & $\mathrm{n}$ & $(\%)$ & $\mathrm{n}$ & $(\%)$ \\
\hline \multicolumn{7}{|l|}{ Gender } \\
\hline Male & 555 & $(100.0)$ & 31 & $(2.2)$ & 667 & $(49.0)$ \\
\hline Female & & & 1,382 & $(97.8)$ & 694 & $(51.0)$ \\
\hline \multicolumn{7}{|l|}{ Age group (years) } \\
\hline Younger than 55 & 31 & $(5.6)$ & 422 & $(29.9)$ & 261 & $(19.2)$ \\
\hline $55-64$ & 137 & $(24.7)$ & 519 & $(36.7)$ & 465 & $(34.2)$ \\
\hline $65-74$ & 114 & $(20.5)$ & 218 & $(15.4)$ & 262 & $(19.3)$ \\
\hline 75 or older & 273 & $(49.2)$ & 254 & $(18.0)$ & 373 & $(27.4)$ \\
\hline \multicolumn{7}{|l|}{ Geographic region } \\
\hline Northeast & 58 & $(10.5)$ & 146 & $(10.3)$ & 120 & $(8.8)$ \\
\hline North central & 166 & $(29.9)$ & 421 & $(29.8)$ & 485 & $(35.6)$ \\
\hline South & 195 & $(35.1)$ & 569 & $(40.3)$ & 504 & $(37.0)$ \\
\hline West & 132 & $(23.8)$ & 268 & $(19.0)$ & 248 & $(18.2)$ \\
\hline Unknown & 4 & $(0.7)$ & 9 & $(0.6)$ & 4 & $(0.3)$ \\
\hline \multicolumn{7}{|l|}{ Population density } \\
\hline Urban & 477 & $(85.9)$ & 1,200 & $(84.9)$ & 1,137 & $(83.5)$ \\
\hline Rural & 78 & $(14.1)$ & 213 & $(15.1)$ & 224 & $(16.5)$ \\
\hline \multicolumn{7}{|l|}{ Payer } \\
\hline Commercial & 165 & $(29.7)$ & 932 & $(66.0)$ & 709 & $(52.1)$ \\
\hline Medicare & 390 & $(70.3)$ & 481 & $(34.0)$ & 652 & $(47.9)$ \\
\hline \multicolumn{7}{|l|}{ Discharge status } \\
\hline Home & 324 & $(58.4)$ & 927 & $(65.6)$ & 1,054 & $(77.4)$ \\
\hline Death & 15 & $(2.7)$ & 36 & $(2.5)$ & 19 & $(1.4)$ \\
\hline Skilled nursing/rehabilitation facility & 99 & $(17.8)$ & 247 & $(17.5)$ & 146 & $(10.7)$ \\
\hline Acute care facility & 47 & $(8.5)$ & 88 & $(6.2)$ & 81 & $(6.0)$ \\
\hline Intermediate care or other facility & 8 & $(1.4)$ & 32 & $(2.3)$ & 14 & $(1.0)$ \\
\hline Other & 79 & $(14.2)$ & 212 & $(15.0)$ & 181 & $(13.3)$ \\
\hline \multicolumn{7}{|c|}{$\begin{array}{l}\text { aStudy period was January 1, 2003, through June 30, 2009. Hospitalizations were identified with principal diagnosis (ICD-9-CM) and/or principal procedure (ICD-9-CM } \\
\text { or CPT) codes indicative of local irreversible events defining an SRE. Diagnosis codes were used to identify pathologic fracture, surgery to bone, or spinal cord compression } \\
\text { Procedure codes were used to identify surgery to bone or spinal cord compression (Appendix). } \\
\text { bPatients had } 2 \text { or more claims at least } 30 \text { days apart with primary or secondary diagnosis of multiple myeloma (ICD-9-CM 203.0x), prostate cancer (ICD-9-CM 185.xx, } \\
\text { V10.46), or breast cancer (ICD-9-CM 174.xx, 175.xx, V10.3). Patients with prostate or breast cancer also had at least } 1 \text { non-rule-out bone metastasis diagnosis (ICD-9- } \\
\text { CM 198.5) on or before the date of admission. } \\
\text { CPT=Current Procedural Terminology; ICD-9-CM =International Classification of Diseases, Ninth Revision, Clinical Modification; SD =standard deviation; SRE= skele- } \\
\text { tal-related event. }\end{array}$} \\
\hline
\end{tabular}

secondary diagnosis (ICD-9-CM) and/or secondary procedure (ICD-9-CM or CPT) codes indicative of local irreversible events defining an SRE as selected by a third-party physician with expertise in ICD-9-CM and CPT coding (Figure 1). Diagnosis codes identified pathologic fracture, surgery to bone, or spinal cord compression, and procedure codes identified surgery to bone or spinal cord compression (Appendix). Although radiation to bone is also a local irreversible event, it was not included as an independent SRE in this study of inpatient costs because the procedure is primarily conducted in the outpatient setting. Hypercalcemia of malignancy, another recognized complication of bone metastases, is a systemic and potentially reversible event and was thus not considered to be a component of the
SRE. ${ }^{21}$ Bone pain was similarly not included. Hospitalizations with negative or no reimbursed amounts were excluded from the analysis. In addition, hospitalizations within 30 days of a previous SRE hospitalization discharge date were excluded to avoid counting readmissions resulting from complications from the previous hospitalization.

\section{SRE Identification and Categorization}

When secondary diagnosis or procedure codes for SREs were present in the claim, they were used to more precisely identify the type of SRE for which the patient was treated, as we assumed that, on average, 1 SRE per admission occurred and that the procedure was performed to treat the diagnosis 


\section{Payer Costs for Inpatient Treatment of Pathologic Fracture, Surgery to Bone, and Spinal Cord Compression Among Patients with Multiple Myeloma or Bone Metastasis Secondary to Prostate or Breast Cancer}

identified in the claim. For instance, surgery to bone includes procedures to set or stabilize a fracture or to prevent an imminent fracture or spinal cord compression. We verified our assumption in the breast cancer sample and found that, for all claims with spinal cord compression and surgery codes, the surgery location was to the spine. Similarly, for all claims with spinal cord compression and pathologic fracture codes, the location of the fracture was the vertebrae. For claims with pathologic fracture and surgery codes, the surgery site was consistent with the fracture site in all but 3\% of cases.

Consequently, in cases where both surgery and pathologic fracture codes were present, we assumed that the surgery was to treat the pathologic fracture, and thus the SRE was classified as a pathologic fracture. In cases where both surgery and spinal cord compression codes were present, we assumed that surgery was to the spine and thus categorized the SRE as a spinal cord compression. In cases where both pathologic fracture and spinal cord compression codes were present, we assumed that the location of the fracture was the vertebrae and thus categorized the SRE as a spinal cord compression. This method created 3 mutually exclusive categories: spinal cord compression with or without pathologic fracture and/or surgery to the bone; pathologic fracture with or without surgery to the bone; and only surgery to the bone.

\section{Outcome Measures}

The primary study outcomes were payer costs and length of stay (LOS). Payer cost per SRE-associated hospitalization was measured as reimbursed amount paid by the health plan to a hospital, including the Medicare-paid portion, after subtracting patient copayments and deductibles. SRE-associated hospital costs included reimbursement of all claims for the full hospital stay until discharge for SRE-related hospitalizations as defined above. Start and end dates of service using room and board revenue codes were identified and all services within that duration, including professional services, were attributed to the hospital stay. Emergency room visits that resulted in the direct admission to the hospital were captured as inpatient admission costs. All costs were adjusted to June 2009 dollars using the medical care component of the Consumer Price Index. ${ }^{22}$

LOS per SRE-associated hospitalization was calculated and was assessed by type of cancer and type of SRE.

\section{Statistical Analysis}

Payer costs and LOS for SRE-associated hospitalizations were examined using descriptive analyses that included descriptive profiles of patients' demographic characteristics and hospital discharge status. In bivariate analyses, the distributions of payer cost and LOS per hospitalization were examined by type of cancer and type of SRE. In anticipation of a skewed cost distribution, we planned a priori to conduct a sensitivity analysis excluding extremely low or high costs of hospitalizations
TABLE 2 Hospitalizations by SRE and Tumor Type

\begin{tabular}{|c|c|c|c|}
\hline & $\begin{array}{c}\text { Hospitalizations } \\
\text { with SRE } \\
\text { as Primary } \\
\text { Diagnosis or } \\
\text { Procedure }\end{array}$ & Readmissions & $\begin{array}{c}\text { Final } \\
\text { Sample Size }\end{array}$ \\
\hline $\begin{array}{l}\text { Prostate cancer with } \\
\text { bone metastasis }\end{array}$ & 599 & & 572 \\
\hline Bone surgery alone & $130 \quad(21.7 \%)$ & 8 & $122(21.3 \%)$ \\
\hline Pathologic fracture ${ }^{a}$ & $416 \quad(69.4 \%)$ & 16 & $400 \quad(70.0 \%)$ \\
\hline $\begin{array}{l}\text { Spinal cord } \\
\text { compression }^{\mathrm{a}}\end{array}$ & $53 \quad(8.9 \%)$ & 3 & $50 \quad(8.7 \%)$ \\
\hline $\begin{array}{l}\text { Breast cancer with } \\
\text { bone metastasis }\end{array}$ & 1,629 & & 1,542 \\
\hline Bone surgery alone & $237 \quad(14.5 \%)$ & 6 & $231 \quad(15.0 \%)$ \\
\hline Pathologic fracture $^{a}$ & $1,293 \quad(79.4 \%)$ & 75 & $1,218 \quad(79.0 \%)$ \\
\hline $\begin{array}{l}\text { Spinal cord } \\
\text { compression }^{a}\end{array}$ & $99 \quad(6.1 \%)$ & 6 & $93 \quad(6.0 \%)$ \\
\hline Multiple myeloma & 1,598 & & 1,495 \\
\hline Bone surgery alone & $359 \quad(22.5 \%)$ & 6 & $353(23.6 \%)$ \\
\hline Pathologic fracture $^{a}$ & $1,101 \quad(68.9 \%)$ & 80 & $1,021 \quad(68.3 \%)$ \\
\hline $\begin{array}{l}\text { Spinal cord } \\
\text { compression }^{\mathrm{a}}\end{array}$ & $138 \quad(8.6 \%)$ & 17 & $121 \quad(8.1 \%)$ \\
\hline
\end{tabular}

${ }^{a}$ Categorizations are mutually exclusive. Hospitalizations for pathologic fracture are with or without surgery to the bone, and hospitalizations for spinal cord compression are with or without surgery to the bone and/or pathologic fracture. $S R E=$ skeletal-related event

within the upper $1 \%$ and lower $1 \%$ of total costs to estimate their impact on mean payer costs and LOS.

To handle uncertainties in possible small sample sizes for less frequently occurring SREs (e.g., spinal cord compression), CIs for mean costs were estimated by the bootstrap method (repeated resampling with replacement from the original sample). ${ }^{23}$ The benefit of performing bootstrapping is that only one assumption is required, which is that the patients in this study were representative of the typical patient with advanced prostate or breast cancer or myeloma experiencing these events. We randomly selected a new sample of patients (with repeat patient selection possible) for each cohort of cancer and SRE type and estimated mean costs for each. We repeated this sampling 1,000 times to generate 1,000 estimates of mean cost for each cohort of SRE by tumor type. Confidence intervals (95\%) were then constructed based on these 1,000 randomly drawn samples. All descriptive analyses were conducted using SAS version 9.1 (SAS Institute Inc., Cary, NC), and bootstrapping was conducted using Stata 11 (StataCorp, College Station, TX).

\section{Results}

\section{Demographic Characteristics and Discharge Status}

Patient demographic characteristics are reported in Table 1. The mean (SD) ages in years were 72.1 (11.6) for patients with 


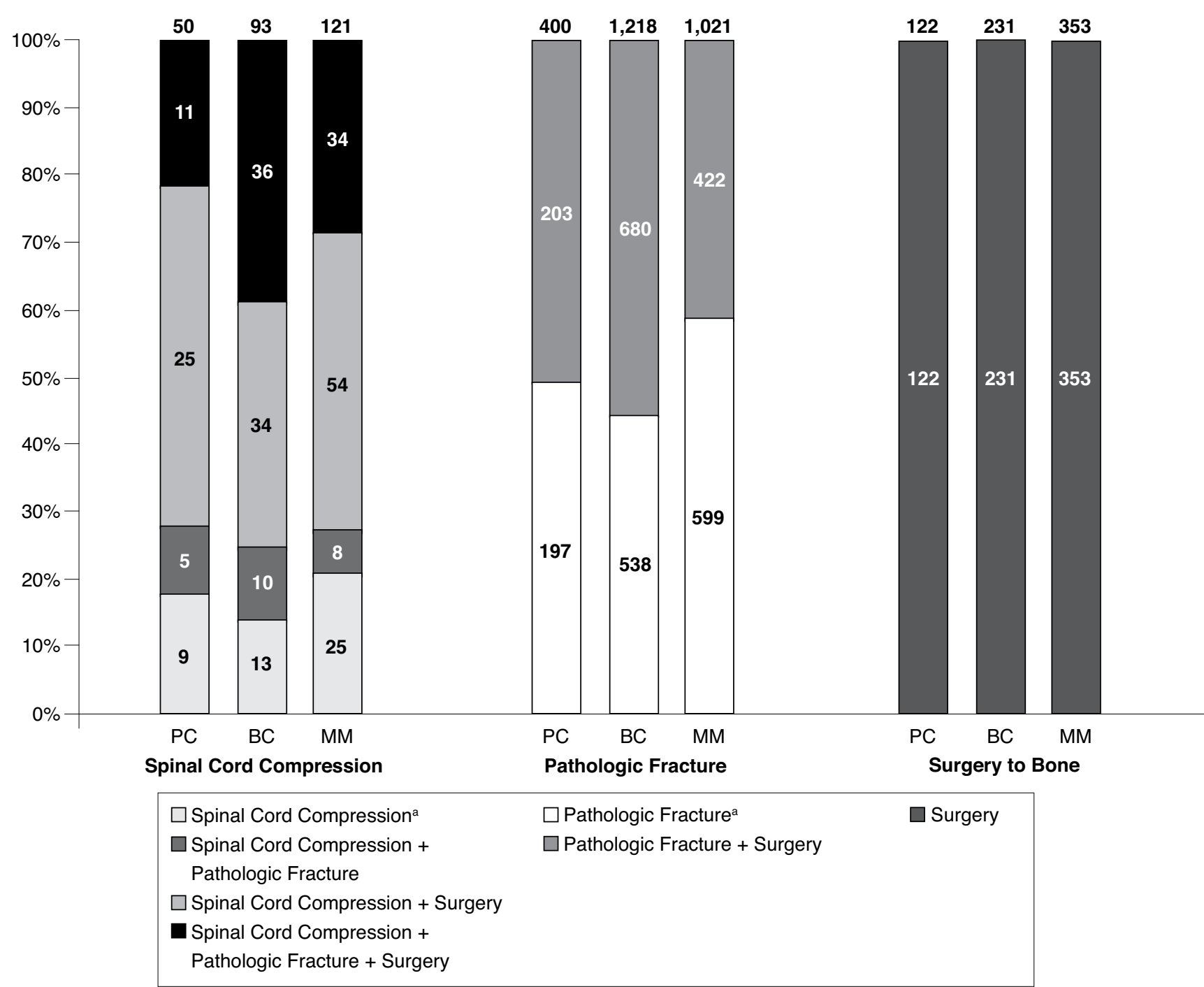

${ }^{a}$ Mutually exclusive categorization: hospitalizations for pathologic fracture are with or without surgery to the bone, and hospitalizations for spinal cord compression are with or without surgery to the bone and/or pathologic fracture.

$B C=$ breast cancer; $M M=$ multiple myeloma; $P C=$ prostate cancer $S R E=$ skeletal-related event .

prostate cancer, 61.3 (12.8) for patients with breast cancer, and 65.4 (12.5) for patients with multiple myeloma. Approximately $70 \%$ of patients with prostate cancer, $33 \%$ of patients with breast cancer, and $47 \%$ of patients with multiple myeloma were 65 years or older. Across all cancer types, between $65 \%$ and $73 \%$ of patients lived in the southern or north central areas of the United States. This distribution reflects the regional distribution of covered lives in the MarketScan database and does not necessarily reflect the regional distribution of SRE-associated admissions in the United States. Across the cancer types, $58 \%-77 \%$ of patients were discharged to home, $11 \%-18 \%$ to rehabilitation facilities, and 6\%-8\% to acute care facilities. Between 1\% and 3\% of patients died during an SREassociated hospitalization.

A total of 572 hospitalizations for prostate cancer, 1,542 for breast cancer, and 1,495 for multiple myeloma met the inclusion criteria (Figure 1). Between $4.5 \%$ and $6.4 \%(n=217)$ of hospitalizations were excluded from the study across the cancer types because they occurred within 30 days of a previous SRE hospitalization discharge date. For all 3 types of cancer, using 


\section{Payer Costs for Inpatient Treatment of Pathologic Fracture, Surgery to Bone, and Spinal Cord Compression Among Patients with Multiple Myeloma or Bone Metastasis Secondary to Prostate or Breast Cancer}

\section{TABLE 3 Distribution of Health Plan Costs and Hospital Length of Stay for Selected Skeletal-Related Events}

\begin{tabular}{|c|c|c|c|c|c|c|}
\hline & \multicolumn{2}{|c|}{ Surgery to the Bone } & \multicolumn{2}{|c|}{ Pathologic Fracture $^{a}$} & \multicolumn{2}{|c|}{ Spinal Cord Compression ${ }^{\mathrm{a}}$} \\
\hline & Health Plan Cost ${ }^{\mathrm{b}}$ & Hospital LOS & Health Plan Cost ${ }^{b}$ & Hospital LOS & Health Plan Cost ${ }^{b}$ & Hospital LOS \\
\hline \multicolumn{7}{|c|}{ Prostate cancer $^{\mathrm{c}}$} \\
\hline $\mathrm{N}$ & \multicolumn{2}{|c|}{122} & \multicolumn{2}{|c|}{400} & \multicolumn{2}{|c|}{50} \\
\hline Mean [SD] & $\$ 42,094[\$ 70,746]$ & $7.7[9.1]$ & $\$ 22,390[\$ 28,042]$ & $6.6[5.6]$ & $\$ 59,788[\$ 66,466]$ & $11.6[10.4]$ \\
\hline Q1, Q3 & $\$ 10,367, \$ 43,318$ & 3,9 & $\$ 7,582, \$ 24,801$ & 3,8 & $\$ 10,950, \$ 98,643$ & 5,15 \\
\hline Median & $\$ 20,641$ & 5.5 & $\$ 14,052$ & 5 & $\$ 30,047$ & 9 \\
\hline Min-Max & $\$ 1,333-\$ 649,392$ & $1-65$ & $\$ 0-\$ 254,498$ & $1-47$ & $\$ 2,288-\$ 302,681$ & $1-50$ \\
\hline \multicolumn{7}{|c|}{ Breast cancer $^{\mathrm{c}}$} \\
\hline $\mathrm{N}$ & \multicolumn{2}{|c|}{231} & \multicolumn{2}{|c|}{1,218} & \multicolumn{2}{|c|}{93} \\
\hline Mean [SD] & $\$ 32,742[\$ 34,836]$ & $6.1[5.3]$ & $\$ 26,936[\$ 29,727]$ & $6.8[6.2]$ & $\$ 59,854[\$ 59,334]$ & $9.4[8.2]$ \\
\hline Q1, Q3 & $\$ 11,645, \$ 43,734$ & 3,8 & $\$ 11,100, \$ 30,512$ & 3,8 & $\$ 18,767, \$ 76,831$ & 4,11 \\
\hline Median & $\$ 20,269$ & 4 & $\$ 17,505$ & 5 & $\$ 37,170$ & 8 \\
\hline Min-Max & $\$ 1,483-\$ 311,701$ & $1-36$ & $\$ 0-\$ 291,361$ & $1-69$ & $\$ 1,422-\$ 285,944$ & $1-49$ \\
\hline \multicolumn{7}{|c|}{ Multiple myeloma } \\
\hline $\mathrm{N}$ & \multicolumn{2}{|c|}{353} & \multicolumn{2}{|c|}{1,021} & \multicolumn{2}{|c|}{121} \\
\hline Mean [SD] & $\$ 31,016[\$ 31,211]$ & $5.9[7.4]$ & $\$ 23,347[\$ 31,605]$ & $6.2[7.4]$ & $\$ 43,691[\$ 53,986]$ & $7.6[7.6]$ \\
\hline Q1, Q3 & $\$ 11,991, \$ 38,834$ & 2,6 & $\$ 8,056, \$ 25,622$ & 2,8 & $\$ 12,070, \$ 49,516$ & 3,9 \\
\hline Median & $\$ 22,623$ & 4 & $\$ 14,992$ & 4 & $\$ 24,623$ & 5 \\
\hline Min-Max & $\$ 0-\$ 218,337$ & 177 & $\$ 0-\$ 514,798$ & $1-92$ & $\$ 0-\$ 381,481$ & $1-40$ \\
\hline \multicolumn{7}{|c|}{$\begin{array}{l}{ }^{a} \text { Categorizations are mutually exclusive. Hospitalizations for pathologic fracture are with or without surgery to the bone, and hospitalizat } \\
\text { are with or without surgery to the bone and/or pathologic fracture. } \\
\text { bReimbursed amount paid by the health plan, including the Medicare-paid portion, after subtracting patient copayments and deductibles. } \\
\text { 'With metastasis to the bone. } \\
\text { LOS=length of stay; Q1 =quartile } 1 \text { (25th percentile); Q3 =quartile } 3 \text { (75th percentile); SD =standard deviation. }\end{array}$} \\
\hline
\end{tabular}

the mutually exclusive classification method, hospitalization occurred most often for pathologic fracture with or without surgery to bone (Figure 2, Table 2). For prostate cancer, 21.3\% of hospitalizations were categorized as surgery to the bone, $69.9 \%$ as pathologic fracture (with or without surgery to the bone), and $8.7 \%$ as spinal cord compression (with or without pathologic fracture and/or surgery to the bone). In the breast cancer cohort, $15.0 \%$ of hospitalizations were categorized as surgery to the bone, $79.0 \%$ as pathologic fracture, and $6.0 \%$ as spinal cord compression. In the multiple myeloma cohort, $23.6 \%$ of hospitalizations were categorized as surgery to the bone, $68.3 \%$ as pathologic fracture, and $8.1 \%$ as spinal cord compression.

Diagnosis and procedure codes for more than 1 SRE type were present in 244 (42.7\%) of hospitalizations in the prostate cancer group, in 760 (49.3\%) for breast cancer, and in 518 (34.6\%) for multiple myeloma. The proportions of SRE code combinations that occurred under the spinal cord compression and pathologic fracture categories were generally similar across tumor types (Figure 2).

\section{Payer Costs and Length of Stay}

Table 3 reports mean health plan costs and LOS by type of cancer and type of SRE, again using the mutually exclusive categorization method. For patients with prostate cancer, mean (SD) payer costs were $\$ 42,094(\$ 70,746)$ for surgery to the bone, $\$ 22,390$ (\$28,042) for pathologic fracture, and $\$ 59,788$ $(\$ 66,466)$ for spinal cord compression. Mean (SD) LOS per admission was 7.7 (9.1) days for surgery to the bone, 6.6 (5.6) days for pathologic fracture, and 11.6 (10.4) days for spinal cord compressions.

For patients with breast cancer, mean (SD) payer costs were $\$ 32,742(\$ 34,836)$ for surgery to the bone, $\$ 26,936(\$ 29,727)$ for pathologic fracture, and $\$ 59,854(\$ 59,334)$ for spinal cord compression. Mean (SD) LOS was 6.1 (5.3) days for surgery to the bone, 6.8 (6.2) days for pathologic fracture, and 9.4 (8.2) days for spinal cord compressions.

For patients with multiple myeloma, mean (SD) payer costs were $\$ 31,016(\$ 31,211)$ for surgery to the bone, $\$ 23,347$ $(\$ 31,605)$ for pathologic fracture, and $\$ 43,691(\$ 53,986)$ for spinal cord compression. Mean (SD) LOS was 5.9 (7.4) days for surgery to the bone, 6.2 (7.4) days for pathologic fracture, and 7.6 (7.6) days for spinal cord compressions.

After excluding the upper $1 \%$ and lower $1 \%$ of reimbursement costs, mean cost estimates declined across all tumor types, ranging from $\$ 29,516$ to $\$ 36,165$ for surgery to the bone, $\$ 20,855$ to $\$ 25,447$ for pathologic fracture, and $\$ 39,957$ to $\$ 58,012$ for spinal cord compression. For prostate cancer, mean cost estimates decreased by $14.1 \%$ for patients experiencing surgery to the bone, $6.9 \%$ for pathologic fracture, and $6.5 \%$ for spinal cord compression. For breast cancer, mean cost estimates decreased by $6.1 \%$ for surgery to bone, $5.5 \%$ for 


\section{TABLE 4}

Mean Health Plan Costs ${ }^{\mathrm{a}}$ and 95\% Bootstrapped Confidence Intervals for Hospitalizations for Selected SREs by SRE Type and Tumor Type

Mean $(95 \% \mathrm{CI})$

\begin{tabular}{|c|c|c|}
\hline \multicolumn{3}{|l|}{ Surgery to the bone } \\
\hline Prostate cancerb & $\$ 42,094$ & $(\$ 29,247-\$ 54,941)$ \\
\hline Breast cancerb & $\$ 32,742$ & $(\$ 28,417-\$ 37,067)$ \\
\hline Multiple myeloma & $\$ 31,016$ & $(\$ 27,823-\$ 34,210)$ \\
\hline \multicolumn{3}{|l|}{ Pathologic fracture ${ }^{c}$} \\
\hline Prostate cancer ${ }^{b}$ & $\$ 22,390$ & $(\$ 19,506-\$ 25,273)$ \\
\hline Breast cancerb & $\$ 26,936$ & $(\$ 25,248-\$ 28,625)$ \\
\hline Multiple myeloma & $\$ 23,347$ & $(\$ 21,435-\$ 25,260)$ \\
\hline \multicolumn{3}{|c|}{ Spinal cord compression ${ }^{\mathrm{c}}$} \\
\hline Prostate cancerb & $\$ 59,788$ & $(\$ 41,401-\$ 78,176)$ \\
\hline Breast cancerb & $\$ 59,854$ & $(\$ 47,771-\$ 71,937)$ \\
\hline Multiple myeloma & $\$ 43,691$ & $(\$ 34,198-\$ 53,184)$ \\
\hline \multicolumn{3}{|c|}{$\begin{array}{l}\text { Reimbursed amount paid by the health plan, including the Medicare-paid portion, } \\
\text { after subtracting patient copayments and deductibles. } \\
\text { bWith metastasis to the bone. } \\
{ }^{c} \text { Categorizations are mutually exclusive. Hospitalizations for pathologic fracture are } \\
\text { with or without surgery to the bone, and hospitalizations for spinal cord compres- } \\
\text { sion are with or without pathologic fracture and/or surgery to the bone. } \\
C I=\text { confidence interval; SRE= skeletal-related event. }\end{array}$} \\
\hline
\end{tabular}

pathologic fracture, and 3.1\% for spinal cord compression. For multiple myeloma, mean cost estimates decreased by $4.8 \%$ for surgery to bone, $8.0 \%$ for pathologic fracture, and $8.5 \%$ for spinal cord compression.

Table 4 presents the bootstrapped 95\% CIs of mean payer costs per hospitalization (based on all hospitalizations including those with reimbursed costs in the upper $1 \%$ and lower $1 \%$ ).

\section{Discussion}

Bone lesions secondary to advanced malignancies are a common occurrence and can have devastating clinical consequences in patients. Patients with bone lesions can experience burdensome SREs such as pathologic fracture, spinal cord compression, or need for surgery or radiation to bone. These skeletal complications are also important from a health economic perspective in prostate and breast cancers, given the prevalence of these diseases, and in multiple myeloma due to the extent of bone destruction that occurs with the disease.

In this study, we used 2 large national claims databases to estimate the mean costs that third-party payers incur for reimbursing SRE-associated hospitalizations (excluding radiation to bone, hypercalcemia of malignancy, and bone pain) among patients with multiple myeloma or bone metastasis secondary to prostate or breast cancer. Mean hospital reimbursements were higher across tumor types for spinal cord compression with or without pathologic fracture and/or surgery to the bone $(\$ 43,691-\$ 59,854)$ than for surgery to the bone only $(\$ 31,016-\$ 42,094)$ or pathologic fracture with or without surgery to the bone $(\$ 22,390-\$ 26,936)$.

The mutually exclusive categorizations used to classify claims under 1 SRE when more than 1 SRE code was present allowed us to develop a composite view of each SRE, thus better reflecting what is occurring in patients. For example, the composition of spinal cord compression as an SRE allowed up to 4 possible combinations of coding: spinal cord compression only, spinal cord compression plus pathologic fracture, spinal cord compression plus surgery to the bone, or spinal cord compression plus pathologic fracture and surgery to bone. Since each of these 4 possibilities is represented in the spinal cord compression claims selected in our study across tumor types, we are more confident that the average costs calculated here provide a reliable estimate of the inpatient costs.

Lastly, we conducted sensitivity analyses to account for skewed cost distributions and potentially small samples of patients in the subgroups of SRE and cancer type. After removing the outliers, the mean cost estimates did not change substantially for the majority of SREs across tumor types.

\section{Limitations}

First, the study was limited to SREs in inpatient settings only. Results do not represent the cost of SREs overall or of treatments provided in outpatient settings. Moreover, the study patients represent only a portion of patients with SREs because not all patients with SREs are treated in inpatient settings. Second, the results represent net payer cost after subtracting patient copayments and deductibles, rather than total allowed cost. To address the possibility that exclusion of patient paid amounts affected our findings, we analyzed the percentage of costs paid by patients and found that it was small, ranging from $1.5 \%$ to $4.2 \%$ of total cost depending on tumor type and SRE type. Third, a common limitation in studies using administrative health care databases is selecting claims based on ICD-9-CM and CPT codes that accurately represent the specific population of interest and clinical endpoints such as SRE-associated hospitalizations. To minimize the potential bias in selecting claims and improve the specificity for selecting the correct population in our study, we consulted with an independent coding expert to identify a list of codes for extracting SRE-associated hospitalizations for this analysis. Fourth, our analysis is based on a sample of patients covered by health care plans from medium to large employers and some who are also covered by Medicare (dual-eligibility). The results from our study may not reflect the full spectrum of reimbursed amounts for these SRE-associated hospitalizations and might have limited generalizability. Fifth, we assumed that patients identified in this database are typical patients with cancer and bone metastasis. Patient age in these populations is consistent with the SEER database ${ }^{24}$ however, no further comparisons were made. 


\section{Payer Costs for Inpatient Treatment of Pathologic Fracture, Surgery to Bone, and Spinal Cord Compression Among Patients with Multiple Myeloma or Bone Metastasis Secondary to Prostate or Breast Cancer}

\section{Conclusions}

The inpatient costs associated with treating SREs are significant from a payer perspective. The present study used a careful process for patient selection and a mutually exclusive categorization method that assessed heterogeneity among SRE types and tumor types. This study provides an estimate that is helpful to payers in quantifying the inpatient financial impact of cancer-related SREs. SREs place a substantial burden on patients with advanced tumors and on third-party payers.

\section{Authors}

ARIE BARLEV, PharmD, MS, is Senior Manager, Global Health Economics; VIDYA SETTY, MPH, MBA, is Medical Writing Manager, Global Medical Writing; and KAREN CHUNG, PharmD, MS, is Director, Global Health Economics, Amgen Inc., Thousand Oaks, California. XUE SONG, PhD, is Senior Research Leader, Thomson Reuters, Cambridge, Massachusetts. BORIS IVANOV, MS, is Lead Programmer, Thomson Reuters, Washington, DC.

AUTHOR CORRESPONDENCE: Arie Barlev, PharmD, MS, Amgen Inc., One Amgen Center Dr., MS 28-3-A, Thousand Oaks, CA 91320. Tel.: 805.447.9347; Fax: 805.376.1816;

E-mail: abarlev@amgen.com.
4. Berruti A, Dogliotti L, Bitossi R, et al. Incidence of skeletal complications in patients with bone metastatic prostate cancer and hormone refractory disease: predictive role of bone resorption and formation markers evaluated at baseline. J Urol. 2000;164(4):1248-53.

5. Carlin BI, Andriole GL. The natural history, skeletal complications, and management of bone metastases in patients with prostate carcinoma Cancer. 2000;88(12 Suppl):2989-94. Available at: http://onlinelibrary. wiley.com/doi/10.1002/1097-0142(20000615)88:12\%2B\%3C2989::AIDCNCR14\%3E3.0.CO;2-Q/pdf. Accessed October 8, 2010.

6. Coleman RE. Clinical features of metastatic bone disease and risk of skeletal morbidity. Clin Cancer Res. 2006;12(20 Pt 2):6243s-6249s. Available at: http://clincancerres.aacrjournals.org/content/12/20/6243s.full.pdf. Accessed October 8, 2010.

7. Trinkaus M, Simmons C, Myers J, Dranatisaris G, Clemons M. Skeletalrelated events (SREs) in breast cancer patients with bone metastases treated in the nontrial setting. Support Care Cancer. 2010;18(2):197-203.

8. Coleman RE, Guise TA, Lipton A, et al. Advancing treatment for metastatic bone cancer: consensus recommendations from the Second Cambridge Conference. Clin Cancer Res. 2008;14(20):6387-95. Available at: http://clincancerres.aacrjournals.org/content/14/20/6387.full.pdf. Accessed October 8, 2010

9. Lipton A, Theriault RL, Hortobagyi GN, et al. Pamidronate prevents skeletal complications and is effective palliative treatment in women with breast carcinoma and osteolytic bone metastases: long term follow-up of two randomized, placebo-controlled trials. Cancer. 2000;88(5):1082 90. Available at: http://onlinelibrary.wiley.com/doi/10.1002/(SICI)10970142(20000301)88:5\%3C1082::AID-CNCR20\%3E3.0.CO;2-Z/pdf. Accessed October 8, 2010.

10. Saad F, Gleason DM, Murray R, et al. A randomized, placebo-controlled trial of zoledronic acid in patients with hormone-refractory metastatic prostate carcinoma. J Natl Cancer Inst. 2002;94(19):1458-68. Available at: http:// jnci.oxfordjournals.org/content/94/19/1458.full.pdf. Accessed October 8, 2010.

11. Saad F, Gleason DM, Murray R, et al. Long-term efficacy of zoledronic acid for the prevention of skeletal complications in patients with metastatic hormone-refractory prostate cancer. J Natl Cancer Inst. 2004;96(11):87982. Available at: http://jnci.oxfordjournals.org/content/96/11/879.full.pdf Accessed October 8, 2010

12. Vogel CL, Yanagihara RH, Wood AJ, et al. Safety and pain palliation of zoledronic acid in patients with breast cancer, prostate cancer, or multiple myeloma who previously received bisphosphonate therapy. Oncologist. 2004;9(6):687-95. Available at: http://theoncologist.alphamedpress.org/cgi/ reprint/9/6/687. Accessed October 8, 2010.

13. American Cancer Society. Cancer facts and figures 2007. Atlanta: American Cancer Society; 2007. Available at: http://www.cancer.org/acs/ groups/content/@nho/documents/document/caff2007pwsecuredpdf.pdf. Accessed October 8, 2010.

14. Chirgwin JM, Guise TA. Skeletal metastases: decreasing tumor burden by targeting the bone microenvironment. J Cell Biochem. 2007;102(6):133342

15. Novartis Pharmaceuticals Corporation. Zometa (zoledronic acid) prescribing information. Available at: http://www.pharma.us.novartis.com/ product/pi/pdf/Zometa.pdf. Accessed October 8, 2010

16. Henry D, Von Moos RAF, Vadhan-Raj S, et al. A double-blind, randomized study of denosumab versus zoledronic acid for the treatment of bone metastases in patients with advanced cancer (excluding breast and prostate cancer) or multiple myeloma. Eur J Cancer. 2009;7(3):11 [Abstract 20LBA]. Poster presented at: Joint 15th Congress of the European CanCer Organisation and 34th Congress of the European Society for Medical Oncology; September 20-24, 2009; Berlin, Germany.

17. Santini D, Fratto ME, Vincenzi B, et al. Denosumab: the era of targeted therapies in bone metastatic diseases. Curr Cancer Drug Targets. 2009;9(7):834-42. 


\section{Payer Costs for Inpatient Treatment of Pathologic Fracture, Surgery to Bone, and Spinal Cord Compression Among Patients with Multiple Myeloma or Bone Metastasis Secondary to Prostate or Breast Cancer}

18. Stopeck A, Body J, Fujiwara Y, et al. Denosumab versus zoledronic acid for the treatment of breast cancer patients with bone metastases: results of a randomized phase 3 study. Eur J Cancer. 2009;7(3):2 (Abstract 2LBA). Poster presented at: Joint 15th Congress of the European CanCer Organisation and 34th Congress of the European Society for Medical Oncology; September 20-24, 2009; Berlin, Germany.

19. Lage MJ, Barber BL, Harrison DJ, Jun S. The cost of treating skeletal-related events in patients with prostate cancer. Am J Manag Care. 2008;14(5):317-22. Available at: http://www.ajmc.com/media/pdf/ AJMC_08may_Lage317to322.pdf. Accessed October 8, 2010.

20. Delea T, McKiernan J, Brandman J, et al. Retrospective study of the effect of skeletal complications on total medical care costs in patients with bone metastases of breast cancer seen in typical clinical practice. J Support Oncol. 2006;4(7):341-47.
21. Ibrahim A, Scher N, Williams G, et al. Approval summary for zoledronic acid for treatment of multiple myeloma and cancer bone metastases. Clin Cancer Res. 2003;9(7):2394-99. Available at: http://clincancerres.aacrjournals.org/content/9/7/2394.full.pdf+html. Accessed October 12, 2010.

22. U.S. Department of Labor, Bureau of Labor Statistics. Measuring price change for medical care in the CPI. Updated April 12, 2010. Available at: http://www.bls.gov/cpi/cpifact4.htm. Accessed October 8, 2010.

23. Efron B, Tibshirani R. An Introduction to the Bootstrap. New York: Chapman \& Hall; 1993.

24. Surveillance Epidemiology and End Results. Table 1.11: Median age of cancer patients at diagnosis, 2003-2007, by primary cancer site, race and sex. SEER Cancer Statistics Review 1975-2007. National Cancer Institute. Available at: http://seer.cancer.gov/csr/1975_2007/results_single/sect_01_ table.11_2pgs.pdf. Accessed October 8, 2010.

\section{APPENDIX Codes to Identify Selected Inpatient SRES}

\begin{tabular}{l|l|l}
\hline Condition & \multicolumn{1}{|c|}{ ICD-9-CM Codes } & \multicolumn{1}{|c}{ CPT Codes } \\
\hline Pathologic fracture & $733.1 \mathrm{x}$ & \\
\hline Bone surgery & $03.53,78.19,78.10-78.13,78.15-78.17,78.19,78.40-78.43$, & $22325-22328,22520-22522,22532-22632,23515,23615-$ \\
& $78.45-78.47,78.49,78.50-78.53,78.55-78.57,78.59,79.10-$ & $23616,23630,24515-24516,24538,24545-24546,24566$, \\
& $79.12,79.15,79.16,79.19,79.20-79.22,79.25,79.26,79.29$, & $24575,24579,24582,24586,24587,24635,24665,24666$, \\
& $79.30-79.32,79.35,79.36,79.39,79.90-79.92,79.95,79.96$, & $24685,25515,25525-25526,25545,25606-25609,27215-$ \\
& $79.99,81.00,81.02-81.08$ & $27218,27226-27228,27235,27236,27244,27245,27248$, \\
& & $27269,27506,27507,27509,27511-27514,27524,27535-$ \\
& & $27536,27540,27756,27758,27759,27766,27769,27784$, \\
\hline Spinal cord compression & $721.1,721.4,721.41,721.42,721.91,722.7,722.70,722.71-$ & 63050,63051 \\
\hline
\end{tabular}

CPT = Current Procedural Terminology; ICD-9-CM = International Classification of Diseases, Ninth Revision, Clinical Modification; SRE=skeletal-related event. 